\title{
A brief review on the use of antidepressants in palliative care
}

\author{
Wadih Rhondali, ${ }^{1,2}$ Michel Reich, ${ }^{3}$ Marilene Filbet ${ }^{2}$
}

Antidepressants are part of the pharmacological management of depressive and anxiety disorders in oncology. In a palliative situation, several properties can enhance their indications, such as hot flushes induced by hormone therapy and neuropathic pain. For frail patients in the palliative stage, pharmacological interactions, their possible side effects and the modalities of their prescription should always be taken into account. In this review, the different classes of antidepressants that can be used in palliative care are described. These classes are briefly compared in terms of efficacy and safety. Suggestions on the best choice according to other symptoms are proposed.

\section{Introduction}

This review presents an update of the antidepressants that can be useful in palliative medicine. Antidepressant treatment can be used for several indications-antidepressants, anxiolytics or co-analgesics. In this article, we describe their use in palliative cancer care for depression or major depressive disorder only, and we do not describe the entire management of depression, which should always include education and counselling. This information can be found in a recent article proposing guidelines on the management of depression in palliative cancer care. ${ }^{1}$

Depression is a frequent problem among cancer patients and has been estimated to occur with a frequency of approximately 15\% (range 1-77.5\%). ${ }^{2}$ Often underdiagnosed and undertreated, depression reduces quality of life and aggravates physical symptoms, such as pain and fatigue. ${ }^{3}$ Depression has also been reported to reduce adherence with cancer treatment. ${ }^{4}$ Moreover, it is an independent predictive risk factor for cancer related mortality. ${ }^{5}$

A recent review of the literature provided evidence that antidepressants are effective in treating depression for patients in

${ }^{1}$ Department of Palliative Care and Rehabilitation Medicine, University of Texas, MD Anderson Cancer Center, Houston, Texas, USA 2Department of Palliative Care, Centre Hospitalier de Lyon-Sud, Hospices Civils de Lyon, Lyon, France ${ }^{3}$ Psycho-Oncology Team, Centre Oscar Lambret, Lille, France

Correspondence to DrW Rhondali, Department of Palliative Care, Centre Hospitalier de Lyon-Sud, Hospices Civils de Lyon, 165 Chemin du Grand Revoyet, 69495 Pierre-Bénite, France; wadih.rhondali@chu-lyon.fr palliative care. ${ }^{6}$ However, antidepressants are underprescribed in palliative care, probably because of some difficulties in distinguishing 'normal' sadness from major depressive disorder or some reluctance to prescribe another medication for patients already taking several drugs (drug interactions). ${ }^{7}$

Previous studies suggest that for patients with advanced cancer, antidepressants should be reserved for major depressive disorder cases because patients who do not have major depressive disorder will not experience improvement in symptoms, wellbeing or survival. ${ }^{8}$

There are several classes of antidepressant agents: tricyclic antidepressants (TCAs), monoamine oxidase inhibitors (MAOIs), selective serotonin reuptake inhibitors (SSRIs), serotoninnorepinephrine reuptake inhibitors (SNRIs) and atypical antidepressants. We will not describe the MAOIs as their use should be avoided in palliative care because of the associated side effects (dizziness, dry mouth, diarrhoea, difficulty urinating, headache) and the high risk of serotonin syndrome or hypertensive crisis when associated with medications such as methadone, tramadol or carbamazepine. Except for bupropion, all classes of antidepressant produce their effect by acting via monoaminergic mechanisms (serotonin, norepinephrine or both). ${ }^{9}$

\section{Tricyclic antidepressants} (eg, amitriptyline, nortriptyline, doxepin, imipramine)

TCAs inhibit the reuptake of serotonin and inhibit norepinephrine transport in corticolimbic structures. TCAs also inhibit histaminic, cholinergic, and $\alpha_{1}$ and $\alpha_{2}$ adrenergic receptors, leading to some of the common side effects of these drugs, such as dry mouth, constipation, orthostatic hypotension, drowsiness, dizziness and cardiovascular toxicity. TCAs are protein bound and are metabolised primarily in the liver. Wide variations of response to antidepressants have been reported between individuals and those variations are mainly explained by patient characteristics such as age, comorbidities and concomitant medication but also by differences in the genetic variant of the enzyme involved in the metabolism of these drugs.

The recommended dosages of TCAs have been proposed empirically, and a recent Cochrane review reported that lower doses of TCAs (75-100 mg/day or less) are equally effective as standard doses but have fewer side effects. ${ }^{10}$ When a TCA is prescribed as a co-analgesic, the dose is usually lower $(50-75 \mathrm{mg}$ ). The long halflife of these drugs enables a $24 \mathrm{~h}$ dosing schedule (table 1).

In cases of neuropathic pain, severe depression with melancholic features or associated symptoms, TCAs might be considered as firstline treatment, ${ }^{11}$ especially if a parenteral regimen is required (available in France). However, these drugs will not always be appropriate for frail patients, such as those in palliative care, because of the potential for increasing frequency of constipation, confusion and other side effects.

\section{Selective serotonin reuptake inhibitors (eg, sertraline, escitalopram, citalopram, paroxetine, fluoxetine)}

SSRIs inhibit the reuptake of serotonin with a better tolerance than TCAs. ${ }^{12}$ In a recent meta-analysis on depression treatment in physically well patients, sertraline and escitalopram demonstrated the best profiles of acceptability but similar efficacy as other SSRIs and several SNRIs. ${ }^{13}$ There has been no equivalent study comparing types of 


\begin{tabular}{|c|c|c|c|c|c|}
\hline Drug & $\begin{array}{l}\text { Recommended dose } \\
\text { (mg/day) }\end{array}$ & $\begin{array}{l}\text { CYT P450 enzymes involved } \\
\text { in metabolism }\end{array}$ & $\begin{array}{l}\text { Contraindications; all antidepressants are } \\
\text { contraindicated in patients with a history of } \\
\text { hypersensitivity to any component }\end{array}$ & Accessory effects & Half-life \\
\hline Nortriptyline & $25-150$ & \multirow{3}{*}{$1 \mathrm{~A} 2,2 \mathrm{C} 9,2 \mathrm{C} 19,2 \mathrm{D} 6,3 \mathrm{~A} 4$} & \multirow{3}{*}{$\begin{array}{l}\text { Cardiac arrhythmias, myocardial infarction, } \\
\text { glaucoma, urinary retention, prostatic } \\
\text { hypertrophy, use of MAOI in the past } 14 \text { days }\end{array}$} & \multirow{3}{*}{ Sedative, anxiety, pain, insomnia } & $20-100$ \\
\hline Doxepin & $10-300$ & & & & $17 \mathrm{~h}$ \\
\hline Imipramine & $25-200$ & & & & $11-25 \mathrm{~h}$ \\
\hline Sertraline & $50-100$ & $2 \mathrm{~B} 6,2 \mathrm{C} 9,2 \mathrm{C} 19,2 \mathrm{D} 6,3 \mathrm{~A} 4$ & & Anxiety, post-traumatic stress disorder & $26 \mathrm{~h}$ \\
\hline Escitalopram & $10-20$ & $2 \mathrm{C} 19,2 \mathrm{D} 6,3 \mathrm{~A} 4$ & & Anxiety & $27-32 \mathrm{~h}$ \\
\hline Citalopram & $20-60$ & & & & $35 \mathrm{~h}$ \\
\hline Paroxetine & $20-60$ & $206,3 \mathrm{~A} 4$ & & Anxiety, post-traumatic stress disorder & $21 \mathrm{~h}$ \\
\hline Duloxetine & $30-120$ & $2 \mathrm{D} 6,1 \mathrm{~A} 2$ & Concomitant use of MAOIs or SSRIs, for & Pain, hot flushes, fibromyalgia & $8-17 \mathrm{~h}$ \\
\hline Venlafaxine & $37.5-225$ & 206 & duloxetine: chronic alcohol use or & Anxiety, hot flushes & $2.5-7.3 \mathrm{~h}$ \\
\hline Milnacipran & $50-100$ & None & liver disease & Pain, fibromyalgia & $8 \mathrm{~h}$ \\
\hline Agomelatine & $25-50$ & $1 \mathrm{~A} 2 / 2 \mathrm{C} 9$ & Liver impairment & Insomnia & $2.3 \mathrm{~h}$ \\
\hline
\end{tabular}

antidepressants in physical ill or palliative care populations.

The SSRIs, which are metabolised primarily in the liver, have a variety of pharmacokinetic parameters, including protein binding, half-life, active metabolites, elimination rates and the type of CYP enzymes that are responsible for SSRI biotransformation. Fluoxetine and paroxetine are metabolised by cytochrome P450 2D6 and inhibit this pathway, resulting in some drug interactions, such as decreasing analgesic effect of opioids (hydrocodone and oxycodone) or decreasing efficacy and levels of the tamoxifen metabolite, endoxifen. Citalopram, escitalopram and sertraline are metabolised through the 3A4, 2C19 and 2D6 pathways. Respectively, and are less likely to generate negative drug interactions.

The most common side effects reported for SSRIs are nausea, diarrhoea, headache, insomnia, jitteriness, fatigue and sexual dysfunction. The most severe adverse effect is the serotonin syndrome, a potentially life threatening adverse drug reaction caused by excess serotonin in the CNS. A recent case report suggested that the risk of this syndrome is increased for patients using strong opioid medications.. ${ }^{14}$ Recommended doses for SSRIs are summarised in table 1.

\section{Serotonin-norepinephrine reuptake inhibitors (eg, venlafaxine, duloxetine, milnacipran)}

SNRIs work by inhibiting the reuptake of serotonin and norepinephrine. They differ from TCAs by their stronger 5-HT effect. ${ }^{15}$
Compared with SSRIs, the efficacy of SNRIs on neuropathic pain is higher and has been reported to be equivalent to that of TCAs. ${ }^{16}$

Similar to SSRIs, SNRIs are metabolised primarily in the liver and are transformed by cytochrome enzymes. Cytochrome enzymes involved in SNRI biotransformation as well as recommended doses are summarised in table 1.

The most common side effects of SNRIs are headache, somnolence, dizziness, insomnia, nausea, dry mouth, constipation, anorexia, mydriasis, sexual dysfunction and weakness. Because elevated blood pressure can also occur, patients at risk of hypertension and heart disease who are taking SNRIs should have their blood pressure monitored.

\section{Atypical antidepressants (eg, trazodone, mirtazapine, tianeptine, bupropion, agomelatine)}

Trazodone is a serotonin antagonist and reuptake inhibitor. Trazodone also has anxiolytic and hypnotic effects. It has no anticholinergic activity, weak $\alpha_{1}$ adrenergic activity and moderate antihistaminic activity. The main side effects of trazodone are sedation and orthostatic hypotension. Trazodone is empirically used in geriatric patients with agitation and insomnia. ${ }^{17}$ The most common side effects are dry mouth and orthostatic hypotension; priaprism occurs rarely.

Mirtazapine is a tetracyclic antidepressant that acts by increasing norepinephrine and serotonin levels (noradrenergic and specific serotoninergic antidepressants). It is a strong antagonist of histaminic $\left(\mathrm{H}_{1}\right)$ adrenergic and muscarinic receptors. The most common side effects are sedation, constipation, dry mouth, dizziness and weight gain. Mirtazapine was recently reported to be one of the most effective antidepressant drugs for nonphysically ill patients. ${ }^{13}$ In a meta-analysis which compared the efficacy and tolerability of 12 common antidepressants (including SSRIs and SNRIs), mirtazapine had superior efficacy and tolerability. An expert opinion (using a Delphi process) reported mirtazapine as one of the best antidepressants for treating depression in the palliative care setting (along with citalopram), probably because of its accessory effects (eg, appetite stimulant and sedative qualities). ${ }^{18}$

Tianeptine is an antidepressant that probably involves glutamate in its mechanism of action. ${ }^{19}$ It has strong antidepressant and anxiolytic effects with good tolerance (eg, less sedative effect, few anticholinergic and cardiovascular adverse effects). ${ }^{19}$ Tianeptine is metabolised in the liver and has a half-life of only $2.5 \mathrm{~h}$. The recommended dose is 3 tablets per day (morning, midday and evening). It is frequently used for elderly patients. The most reported side effects of tianeptine are dry mouth, constipation, dizziness, drowsiness and insomnia.

Bupropion have a specific mechanism of action; it is not fully understood but it seems to work by binding and inhibiting the dopamine and norepinephrine uptake pumps. ${ }^{2021}$ Bupropion is metabolised in the liver into several active metabolites. The most reported side effects are dizziness, headache, insomnia, nausea and dry mouth, and it is associated with an increased risk 
of seizure. Bupropion has a lower risk than other antidepressants for causing sexual dysfunction and weight gain. There are no studies on its use in cancer patients in palliative care.

Agomelatine is a melatonin (MT) analogue drug with a specific mechanism of action. It acts as an MT1/MT2 melatonergic receptor agonist and as a 5-HT2C antagonist. Because it does not bind to 5-HT2A receptors, agomelatine has a more favourable side effect profile than SSRIs. Agomelatine is metabolised in the liver. A recent review reported a similar efficacy to venlafaxine and sertraline, and a higher efficacy than fluoxetine. ${ }^{22}$ There are no studies on its use in cancer patients in palliative care.

\section{Psychostimulants (eg, methylphenidate, modafinil)}

For patients with a short life expectancy, depression can be managed with psychostimulants, which take effect quickly (within 24-48 h). Psychostimulants are reported to be generally well tolerated although common side effects include agitation, confusion and insomnia. ${ }^{11} 23$ Clinicians should be alert to cognitive disturbances such as delirium. In cases of severe depression, patients with longer life expectancy can benefit from starting a psychostimulant while waiting for a prescribed antidepressant agent to be fully effective (4-6 weeks).

\section{Efficacy}

Among the antidepressant drugs available, mirtazapine, escitalopram, venlafaxine and sertraline have been reported as the most effective for physically well patients. ${ }^{13}$ For palliative cancer care, a recent literature review reported that there was still not enough evidence to answer the question of which antidepressant clinicians should choose for their patients. ${ }^{6}$

All antidepressants have a delayed onset of clinical effect (4-6 weeks) but they are not equivalent regarding their accessory effects or functions. ${ }^{6}$ The side effects should be assessed in the first week of treatment and depression should be reassessed (eventually using a specific tool) every 2 weeks. Regarding the assessment, choice of antidepressant in palliative care should be dictated by the patient's symptoms, presented by the patient (eg, pain, poor appetite, insomnia, hot flushes, etc), as well as the potential toxicity. ${ }^{6}$ Furthermore, patients with limited life expectancy (less than 4 weeks) should probably received psychostimulants rather than antidepressants.
Tolerability and safety

In the general population, as for cancer patients, SSRIs are reported to be safer and better tolerated than TCAs, especially by the elderly. ${ }^{624}$ However, the review from Rayner et al showed little difference between the two classes of drug regarding adverse events. ${ }^{6}$ TCAs are associated with a higher risk of dry mouth, blurred vision, dizziness, constipation and tremor whereas SSRIs are associated with a higher frequency of nausea, diarrhoea, insomnia and headache. ${ }^{25}$

\section{Clinical practice propositions}

Treatment should be started as soon as the diagnosis of major depressive disorder is made. The patient should be regularly reassessed and treatment efficacy should be assessed at least 4 weeks after initiation of the antidepressants (a first assessment can be done at 2 weeks looking for a partial response). In the case of a partial or no response at 4-6 weeks of antidepressant medication at an adequate dosage, increasing the dose should be considered. Cases of non-response should be discussed on a case by case basis (switching to an antidepressant from a different class, switching to another antidepressant from the same class or combining antidepressants from different classes). Treatment should be continued for 6-9 months.

Palliative care patients often develop multiple severe symptoms. They are more likely than patients not in palliative care to present with depression associated with symptoms such as pain and fatigue. Thus firstline treatment should be chosen after considering not only the associated symptoms but also the potential side effects.

If a patient presents with both depression and anxiety, SSRIs such as citalopram, escitalopram and paroxetine or NSRIs such as venlafaxine should be favoured.

Venlafaxine has been reported to be effective in treating depression that has not previously responded to SSRIs. Agomelatine and mirtazapine are favoured for cases of insomnia, mirtazapine for anorexia or nausea, mirtazapine and fluoxetine for fatigue, duloxetine and milnacipran for pain, venlafaxine for hot flushes and nortriptyline for agitation or suicidal ideation. In cases of severe liver failure, tianeptine or milnacipran can still be used.

In the case of severe liver failure, we can still use tianeptine or milnacipran. Venlafaxine has been reported to be effective in treating depression that has not previously responded to SSRIs.

\section{Conclusion}

Antidepressant prescription must follow a logical and rational protocol and cannot be

\section{Key message}

Antidepressant drugs have a place in palliative care and the best effectiveness will be achieved by experts who know their pharmacology, clinical effects, side effects and contraindications in this category of patients.

prescribed without a thorough assessment of the medical and psychosocial situation, especially in the palliative care setting. The patient's comorbidities, potential pharmacological interactions and benefittoxicity ratio must be taken into account. Antidepressants, prescription, if well prescribed, can alleviate patient suffering and, to optimise efficiency, should be used in combination with psychotherapeutic interventions.

Contributors WR, MR and MF: writing and revision of the manuscript.

\section{Competing interests None.}

Provenance and peer review Commissioned; externally peer reviewed.

\section{References}

1. Rayner L, Price A, Hotopf M, et al. The development of evidence-based European guidelines on the management of depression in palliative cancer care. Eur J Cancer 2011;47:702-12.

2. Mitchell AJ, Chan M, Bhatti H, et al. Prevalence of depression, anxiety, and adjustment disorder in oncological, haematological, and palliative-care settings: a meta-analysis of 94 interview-based studies. Lancet Oncol 2011;12:160-74.

3. Lloyd-Williams M, Dennis M, Taylor F. A prospec tive study to determine the association between physical symptoms and depression in patients with advanced cancer. Palliat Med 2004;18:558-63.

4. Holland JC, Alici Y. Management of distress in cancer patients. J Support Oncol 2010;8:4-12.

5. Satin JR, Linden W, Phillips MJ. Depression as a predictor of disease progression and mortality in cancer patients: a meta-analysis. Cancer 2009;115:5349-61.

6. Rayner L, Price A, Evans A, et al. Antidepressants for the treatment of depression in palliative care: systematic review and meta-analysis. Palliat Med 2011:25:36-51.

7. Lloyd-Williams M, Friedman T, Rudd N. A survey of antidepressant prescribing in the terminally ill. Palliat Med 1999;13:243-8.

8. Stockler MR, O'Connell R, Nowak AK, et al. Effect of sertraline on symptoms and survival in patients with advanced cancer, but without major depression: a placebo-controlled double-blind randomised trial. Lancet Oncol 2007;8:603-12.

9. Blier P, Abbott FV. Putative mechanisms of action of antidepressant drugs in affective and anxiety disorders and pain. J Psychiatry Neurosci 2001;26:37-43.

10. Furukawa T, McGuire H, Barbui C. Low dosage tricyclic antidepressants for depression. Cochrane Database Syst Rev 2003;3:CD003197.

11. Perry PJ. Pharmacotherapy for major depression with melancholic features: relative efficacy of tricyclic versus selective serotonin reuptake inhibitor antidepressants. J Affect Disord 1996;39:1-6.

12. Rayner L. Antidepressants for depression in physically ill people. Cochrane Database Syst Rev 2010;3:CD007503.

13. Cipriani A, Furukawa TA, Salanti G, et al. Comparative efficacy and acceptability of 12 new- 
generation antidepressants: a multiple-treatments meta-analysis. Lancet 2009;373:746-58.

14. Reich M, Lefebvre-Kuntz D. Serotoninergic antidepressants and opiate analgesics: a sometimespainful association: a case report. Encephale 2010;36 (Suppl 2):D119-23.

15. Roseboom PH, Kalin NH. Neuropharmacology of venlafaxine. Depress Anxiety 2000;12 (Suppl 1):20-9.

16. Selph S. Carson S, Fu R, et al. Drug Class Review: Neuropathic Pain: Final Update 1 Report [Internet]. Portland, OR: Oregon Health \& Science University, 2011.

17. Mukherjee PK, Davey A. Differential dosing of trazodone in elderly depressed patients: a study to investigate optimal dosing. J Int Med Res 1986;14:279-84.
18. Rayner L. Expert opinion on detecting and treating depression in palliative care: a Delphi study. BMC Palliat Care 2011;10:10.

19. McEwen BS, Chattarji S, Diamond DM, et al. The neurobiological properties of tianeptine (Stablon): from monoamine hypothesis to glutamatergic modulation. Mol Psychiatry 2010;15:237-49.

20. Ascher JA, Cole JO, Colin JN, et al. Bupropion: a review of its mechanism of antidepressant activity. J Clin Psychiatry 1995;56:395-401.

21. Foley KF, DeSanty KP, Kast RE. Bupropion: pharmacology and therapeutic applications. Expert Rev Neurother 2006;6:1249-65.

22. Demyttenaere K. Agomelatine: a narrative review. Eur Neuropsychopharmacol 2011;21 (Suppl 4):S703-9.
23. Candy M, Jones L, Williams R, et al. Psychostimulants for depression. Cochrane Database Syst Rev 2008:2:CD006722.

24. Furukawa TA, McGuire H, Barbui C. Metaanalysis of effects and side effects of low dosage tricyclic antidepressants in depression: systematic review. BMJ 2002;325:991.

25. Snow V, Lascher S, Mottur-Pilson C. Pharmacologic treatment of acute major depression and dysthymia. American College of PhysiciansAmerican Society of Internal Medicine. Ann Intern Med 2000;132(9):738-42. 